\title{
PHLST5: A Practical and Improved Version of Polyharmonic Local Sine Transform
}

\author{
Jucheng Zhao, Naoki Saitoł, Yi Wang ${ }^{\ddagger}$
}

\begin{abstract}
We introduce a practical and improved version of the Polyharmonic Local Sine Transform (PHLST) called PHLST5. After partitioning an input image into a set of rectangular blocks, the original PHLST decomposes each block into a polyharmonic component and a residual. Each polyharmonic component solves a polyharmonic equation with the boundary conditions that match the values and normal derivatives of even orders along the boundary of the corresponding block with those of the original image block. Thanks to these boundary conditions, the residual component can be expanded into a Fourier sine series without facing the Gibbs phenomenon, and its Fourier sine coefficients decay faster than those of the original block. Due to the difficulty of estimating normal derivatives of higher orders, however, only the harmonic case (i.e., Laplace's equation) has been implemented to date, which was called Local Laplace Sine Transform (LLST). In that case, the Fourier sine coefficients of the residual decay in the order $O\left(\|\boldsymbol{k}\|^{-3}\right)$, where $\boldsymbol{k}$ is the frequency index vector. Unlike the original PHLST, PHLST5 only imposes the boundary values and the first order normal derivatives as the boundary conditions, which can be estimated using the information of neighbouring image blocks. In
\end{abstract}

*Formerly Department of Mathematics, University of California, Davis, CA 95616 USA. Currently Prescio Consulting, LLC., Casa Grande, AZ 85230 USA.

${ }^{\dagger}$ Department of Mathematics, University of California, Davis, CA 95616 USA.

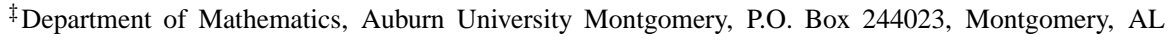
36124 USA. 
this paper, we derive a fast algorithm to compute a 5th degree polyharmonic function that satisfies such boundary conditions. Although the Fourier sine coefficients of the residual of PHLST5 possess the same decaying rate as in LLST, by using additional information of first order normal derivative from the boundary, the blocking artifacts are largely suppressed in PHLST5 and the residual component becomes much smaller than that of LLST. Therefore PHLST5 provides a better approximation result. We shall also show numerical experiments that demonstrate the superiority of PHLST5 over the original LLST in terms of the efficiency of approximation.

keywords: local Fourier analysis, polyharmonic equation, discrete sine transform, image approximation.

\section{Introduction}

One of us (NS) recently introduced the Polyharmonic Local Sine Transform (PHLST) $[18,19]$ in an attempt to develop a local Fourier analysis and synthesis method without encountering the infamous Gibbs phenomenon. The PHLST is also to resolve several problems occurring in the Local Trigonometric Transforms (LTTs) of Coifman and Meyer [7] and Malvar [14, 13], such as the overlapping windows and the slopes of the bell functions. PHLST first segments a given function (or input data) $f(\boldsymbol{x}), \boldsymbol{x} \in \Omega \subset$ $\mathbb{R}^{d}$ supported on an open and bounded domain $\Omega$ into a set of disjoint blocks $\left\{\Omega_{j}\right\}$ such that

$$
\bar{\Omega}=\bigcup_{j=1}^{J} \bar{\Omega}_{j} .
$$

Denote by $f_{j}$ the restriction of the function $f$ to $\bar{\Omega}_{j}$, i.e., $f_{j}=\chi_{\bar{\Omega}_{j}} f$, where $\chi_{\bar{\Omega}_{j}}$ is the characteristic function on the set $\bar{\Omega}_{j}, j=1,2, \cdots, J$. Then PHLST decomposes each $f_{j}$ into two components as $f_{j}=u_{j}+v_{j}$. The components $u_{j}$ and $v_{j}$ are referred to as the polyharmonic component and the residual, respectively. The polyharmonic component is obtained by solving the following polyharmonic equation:

$$
\Delta^{m} u_{j}=0 \quad \text { in } \Omega_{j}, \quad m \in \mathbb{N}
$$


with a set of given boundary values and normal derivatives

$$
\frac{\partial^{q_{\ell}} u_{j}}{\partial \nu^{q_{\ell}}}=\frac{\partial^{q_{\ell}} f}{\partial \nu^{q_{\ell}}} \quad \text { on } \partial \Omega_{j}, \quad \ell=0, \ldots, m-1,
$$

where $\Delta=\sum_{i=1}^{d} \partial^{2} / \partial x_{i}^{2}$ is the Laplace operator in $\mathbb{R}^{d}$. The natural number $m$ is called the degree of polyharmonicity, and $q_{\ell}$ is the order of the normal derivative. These boundary conditions (2) enforce that the solution $u_{j}$ interpolates the function values and the normal derivatives of orders $q_{1}, \ldots, q_{m-1}$ of the original signal $f$ along the boundary $\partial \Omega_{j}$. The parameter $q_{0}$ is normally set to 0 , which means that $u_{j}=f$ on the boundary $\partial \Omega_{j}$, i.e., the Dirichlet boundary condition. If the blocks $\Omega_{j}, j=$ $1,2, \cdots, J$, are all rectangles (of possibly different sizes), PHLST sets $q_{\ell}=2 \ell$, i.e., only normal derivatives of even orders are interplolated. It is not necessary to match normal derivatives of odd orders when the blocks $\Omega_{j}$ 's are rectangular domains. This is because the Fourier sine series of the residual $v_{j}$ is equivalent to the complex Fourier series expansion of the extended $v_{j}$ after odd reflection with respect to the boundary $\partial \Omega_{j}$, hence the continuity of the normal derivatives of odd orders (up to order $2 m-1$ ) is automatically guaranteed. Recall that for $m=1,2$, the equation (1) is usually called Laplace's equation and the biharmonic equation, respectively. We remark that in the case of the domain $\Omega \in \mathbb{R}$ being an interval (i.e., the spatial dimension $d=1$ ), the polyharmonic component $u_{j}$ for $m=1$ is simply a straight line connecting the two boundary points of the interval $\bar{\Omega}_{j}$; while for $m=2$, the polyharmonic component $u_{j}$ is a cubic polynomial connecting the two boundary points. However, when the spatial dimension is higher (i.e., $d \geq 2$ ), the solution of the equation (1) with the boundary conditions (2) is not a simple tensor product of algebraic polynomials in general. Subtracting such $u_{j}$ from $f_{j}$ gives us the residual $v_{j}=f_{j}-u_{j}$ satisfying

$$
\frac{\partial^{q_{\ell}} v_{j}}{\partial \nu^{q_{\ell}}}=0 \quad \text { on } \partial \Omega_{j}, \quad \ell=0, \ldots, m-1
$$

This implies that the values and the normal derivatives of $v_{j}$ vanish on the boundary $\partial \Omega_{j}$. Thus the Fourier sine expansion coefficients of the residual $v_{j}$ decay rapidly, i.e., in the order $O\left(\|\boldsymbol{k}\|^{-2 m-1}\right)$, provided that there is no other intrinsic singularity in 
the domain $\Omega_{j}$, where $k$ is the frequency index vector. In fact, we have the following theorem.

Theorem 1.1. [19] Let $\Omega_{j}$ be a bounded rectangular domain in $\mathbb{R}^{d}$, and let $f_{j} \in$ $C^{2 m}\left(\bar{\Omega}_{j}\right)$, but non-periodic. Assume further that $\left(\partial / \partial x_{i}\right)^{2 m+1} f, i=1, \ldots, d$, exist and are of bounded variation. Furthermore, let $f_{j}=u_{j}+v_{j}$ be the PHLST representation, i.e., the polyharmonic component $u_{j}$ is the solution of the polyharmonic equation (1) of degree $m$ satisfying the boundary conditions (2) with $q_{\ell}=2 \ell$, $\ell=0,1, \ldots, m-1$, and $v_{j}=f_{j}-u_{j}$ is the residual component. Then, the Fourier sine coefficient $b_{\boldsymbol{k}}$ of the residual $v_{j}$ is of order $O\left(\|\boldsymbol{k}\|^{-2 m-1}\right)$ for all $\boldsymbol{k} \neq \mathbf{0}$, where the frequency index vector $\boldsymbol{k}=\left(k_{1}, \ldots, k_{d}\right) \in \mathbb{Z}_{+}^{d}$, and $\|\boldsymbol{k}\|$ is the Euclidean (i.e., $\ell^{2}$ ) norm of $\boldsymbol{k}$.

The proof of this theorem can be found in the paper [19]. We named this way of decomposing a function $f$ into a set of functions $\left\{f_{j}=u_{j}+v_{j}\right\}_{j=1}^{J}$ the Polyharmonic Local Sine Transform (PHLST) with degree of polyharmonicity $m$. Note that if we employ the complex Fourier series expansion or the Fourier sine expansion of non-periodic $f_{j}$ by brute-force periodization, the decaying rate becomes only of order $O\left(\|\boldsymbol{k}\|^{-1}\right)$ even if $f_{j} \in C^{2 m}\left(\bar{\Omega}_{j}\right)$. If we use the Fourier cosine series expansion of $f_{j}$ (as adopted in the JPEG standard), we can obtain a decaying rate of order $O\left(\|\boldsymbol{k}\|^{-2}\right)$. Thus the faster decay of the Fourier sine coefficients of $v_{j}$ in PHLST allows us to distinguish intrinsic singularities in the data from the artificial discontinuities created by local windowing or periodization. It also enables us to interpret the frequency contents of each block without being influenced by the surrounding blocks and without the edge effect such as the Gibbs phenomenon. Moreover, as long as the boundary data are stored and the normal derivatives at the boundary are available, the polyharmonic components can be computed quickly by utilizing the FFT-based Laplace solver developed by Averbuch, Braverman, Israeli, and Vozovoi [1, 4], which we shall call the ABIV method. Combining the fast solver ABIV with the quickly decaying Fourier sine coefficients of the residuals, the usefulness of PHLST to image approximation when the 
degree of polyharmonicity $m=1$ was demonstrated in the papers $[18,19]$. We remark that to remove boundary artifacts, researchers in the field of image processing have proposed several approaches, such as replication edge extension, windowed extrapolation, constant extension, reflection edge extension. A nice overview can be found in the reference [8].

Soon after developing PHLST, N. Saito and K. Yamatani extended it to the Polyharmonic Local Cosine Transform (PHLCT) [23]. The PHLCT allows the Fourier cosine coefficients of the residual decay in the order $O\left(\|\boldsymbol{k}\|^{-2 m-2}\right)$ by setting $q_{\ell}=2 \ell+1$, $\ell=0, \ldots, m-1$ in the boundary conditions (2) and by introducing an appropriate source term on the right hand side of the polyharmonic equation (1). In that work, an efficient algorithm was developed to improve the quality of images already severely compressed by the popular JPEG standard, which is based on Discrete Cosine Transform (DCT).

Finally, N. Saito introduced the Polyharmonic Local Fourier Transform (PHLFT) [19] by setting $q_{\ell}=\ell, \ell=0, \ldots, m-1$ in Eq. (2) and by replacing the Fourier sine series with the complex Fourier series in expanding the $v_{j}$ components. With some sacrifice of the decay rate of the expansion coefficients, i.e., of order $O\left(\|\boldsymbol{k}\|^{-m-1}\right)$ instead of order $O\left(\|\boldsymbol{k}\|^{-2 m-1}\right)$ or of order $O\left(\|\boldsymbol{k}\|^{-2 m-2}\right)$, PHLFT allows one to compute local Fourier magnitudes and phases without facing the Gibbs phenomenon. PHLFT also can capture the important information of orientation much better than PHLST and PHLCT. Moreover, it is fully invertible and should be useful for various filtering, analysis, and approximation purposes.

In all of the above transforms, however, we have only implemented and tested the harmonic case, i.e., the degree of polyharmonicity $m=1$. In other words, in practice, we have only used Laplace's equation as the polyharmonic equation in (1) so far. Consequently, we only demonstrated the decaying rates of PHLST, PHLCT, and PHLFT as $O\left(\|\boldsymbol{k}\|^{-3}\right), O\left(\|\boldsymbol{k}\|^{-4}\right)$, and $O\left(\|\boldsymbol{k}\|^{-2}\right)$, respectively. We call these transforms with polyharmonicity $m=1$ Laplace Local Sine Transform (LLST), Laplace Local Cosine Transform (LLCT), and Laplace Local Fourier Transform (LLFT), re- 
spectively. It is theoretically possible to solve the polyharmonic equation of higher degree of polyharmonicity (i.e., $m>1$ ). But in practice, images are discontinuous almost everywhere and contain noises. The main difficulty is to reliably estimate the required normal derivatives of higher orders at the boundary of each block $\Omega_{j}$. If one tries to use boundary derivatives of higher orders (e.g., those estimated by a higher degree polynomial fit), then their values tend to be chaotically huge. Consequently not only the polyharmonic solution (i.e., the $u$ component) becomes huge compared to the original data, the residual component $v$ is also with large energy. Although the Fourier sine coefficients of the residual $v$ decay rapidly, it is virtually useless for the purpose of approximation. Therefore, in practice we shall not only seek fast decaying rate of the Fourier sine coefficients of the residual $v$, but also a residual $v$ with a small energy. In this paper, we explore a different aspect of PHLST. Instead of blindly seeking fast decaying rate, we consider the polyharmonic equation as a tool to achieve smooth approximation. Assuming the boundary derivatives are accurately estimated, it is clear that the more information of boundary derivatives is used, the better the prediction of the original image from the $u$ component will be. If equally spaced samples are taken from a smooth function, it is well known that a cubic spline approximation always converges to the original function faster than a piecewise linear approximation as the size of the sampling mesh converges to zero. Similarly, we seek a tool which is a higher order approximant than LLST in this paper. In practice, the higher order PHLST can be regarded as an improvement of LLST by reducing both blocking artifacts and the energy of the residual component. We also introduce a practical algorithm to compute PHLST with a 5th degree polyharmonic equation $(m=5)$, yet constrained only by the Dirichlet and Neumann conditions, i.e., by using only $q_{0}=0$ and $q_{1}=1$ in Eq. (2). Therefore, we shall name this method PHLST5. We believe that this is the limit of the practicality in the line of PHLST of higher degree polyharmonicity.

In order to understand our method better, we also compare it with other polyharmonicrelated work. In particular we compare PHLST5, LLST with an approximation method using radial basis functions $[9,10,11,17,6,20]$. Radial basis functions are extremely 
useful when interpolating scattered data, especially of high dimensions. Let $S$ be the set of distinct points in $\mathbb{R}^{d}$, which are traditionally called centers in radial basis function jargon, as radial basis functions are radially symmetric about the centers. The goal is to approximate an unknown smooth function $f$ that is only given at those centers via a set of real values $f_{s}, s \in S$. In order to approximate the unknown function $f$ with an approximant $\tilde{f}$, a univariate continuous function $\phi: \mathbb{R}_{+} \rightarrow \mathbb{R}$ that is radialized by composition with the Euclidean norm is chosen as the radial basis function. Additionally, the centers $s \in S$ are used to shift the radial basis function and as collocation points. Therefore the standard radial function approximants have the form

$$
\tilde{f}(\boldsymbol{x})=\sum_{\boldsymbol{s} \in S} c_{\boldsymbol{s}} \phi(\|\boldsymbol{x}-\boldsymbol{s}\|), \quad \boldsymbol{x} \in \mathbb{R}^{d} .
$$

We remark that when the radial function is chosen as a multiquadric, that is,

$$
\phi(r)=\sqrt{r^{2}+c^{2}},
$$

where $c$ is a positive parameter, the interpolation requirements

$$
\left.\tilde{f}\right|_{S}=\left.f\right|_{S}
$$

for given data $\left.f\right|_{S}$ lead to a nonsingular interpolation matrix

$$
A=\{\phi(\|s-t\|)\}_{s, t \in S}
$$

due to Micchelli [16].

The paper is organized as follows. Section 2 describes the details of how to construct our new transform, PHLST5. Section 3 shows the results of our numerical experiments and demonstrates the improvements of PHLST5 over LLST in terms of the efficiency of approximation. We also compare the performance of PHLST5 with that of radial basis function transform. Finally, we conclude this paper in Section 4 with our discussion of some potential problems and our future plans. In Appendix A, we review the ABIV method for readers' convenience. 


\section{Construction of PHLST5}

In this section, we shall only deal with two-dimensional images (i.e., $d=2$ ), and focus on the analysis of one image block $\Omega_{j}$ for a particular $j$. Therefore, for simplicity, we shall drop the subscript $j$ that was used in many equations appeared in Introduction. Furthermore, we shall assume $\Omega=(0,1)^{2}$, the unit square in $\mathbb{R}^{2}$.

\subsection{Difficulties in Solving a Biharmonic Equation}

Let $f(x, y),(x, y) \in \bar{\Omega}$ be a given input image. If we view LLST to be an approximant to match the intensity values on the boundary, then a natural way to improve LLST is to consider a biharmonic equation

$$
\Delta^{2} u=0 \quad(x, y) \in \Omega,
$$

with boundary condition

$$
\left\{\begin{array}{rlrl}
u & =f & & (x, y) \in \partial \Omega \\
\frac{\partial u}{\partial \nu} & =\frac{\partial f}{\partial \nu} & & (x, y) \in \partial \Omega
\end{array} .\right.
$$

A solution of such a biharmonic system, compared with LLST, guarantees that the regularity of the solution $u$ across the boundary is one order higher. In addition, a biharmonic solution has the minimum curvature property which minimizes the oscillation impact from the boundary data [21]. Theoretically, one can even consider polyharmonic equations of higher order (i.e., $m>2$ ) with given boundary data (i.e., $\left.\left\{\partial^{\ell} f / \partial \nu^{\ell}\right\}_{\ell=0}^{m-1}\right)$. One should notice that the normal derivatives of various orders on the boundary must be estimated from the given original image samples. However, in practice, the estimated values of normal derivative $\partial^{\ell} f / \partial \nu^{\ell}$ with $\ell \geq 2$ are fragile and inaccurate, especially when the original image contains noises. Therefore, any method requires normal derivatives of higher order $(m \geq 2)$ is impractical.

Even if we have accurate estimates of the required nomal derivtives, solving this biharmonic system (4), (5) is numerically difficult. There are quite a few methods to 
numerically solve a biharmonic system. Some representative methods include the finite difference (FD) or FFT-based solver with the FD approximation of the Laplace operator [3]; a method that converts it to an integral equation and iteratively solves the resulting linear system [15]; and the spectral methods based on the Chebyshev or Legendre polynomial expansions [2]. None of them are suitable for our problem though. The FD-based methods have low accuracy in the computed solutions in general. Moreover, both the FD-based methods and the one using the technique of integral equations are computationally expensive. The spectral methods using the Chebyshev or Legendre polynomial expansions require function values sampled on special grids (i.e., the Chebyshev or the Legendre nodes), which are usually not available for our problem because most digital images are sampled on regular rectangular grids.

On the other hand, we wish to retain the flavor of the ABIV method $[1,4]$ as much as possible. For solving Laplace's equation with the Dirichlet boundary condition on a rectangular domain, the ABIV method is ideal because: 1) it is computationally fast due to its use of the FFT algorithm; 2) it is very accurate; 3) the analytical solution provided by the method allows us to interpolate at any point within the domain. See Appendix A for more about the ABIV method. Unfortunately, the biharmonic equation (4) with the boundary conditions (5) does not permit us to use the ABIV method directly.

\subsection{Relaxing the Biharmonic System}

Instead of directly solving the biharmonic system (4), (5), we relax the polyharmonicity (i.e., $m>2$ ) and propose the following polyharmonic system so as to fully incorporate the ABIV method.

$$
\left\{\begin{aligned}
\Delta^{m} u & =0 & & (x, y) \in \Omega \\
u & =f & & (x, y) \in \partial \Omega \\
\frac{\partial u}{\partial \nu} & =\frac{\partial f}{\partial \nu} & & (x, y) \in \partial \Omega .
\end{aligned}\right.
$$

Although we now lose the minimum curvature property, we still have the regularity improvement across the boundary. Note that the system (6) cannot be solved 
uniquely because the boundary conditions make the problem underdetermined. Instead of solving the system (6) directly, we shall explicitly find a polyharmonic function that satisfies the equations in the system (6).

In order to have a proper choice of $m$, we shall decouple the system (6) into two subsystems by representing $u$ in terms of its two components $u_{1}$ and $u_{2}$ (i.e., $u=$ $\left.u_{1}+u_{2}\right)$. The component $u_{1}$ is the solution of Laplace's equation with the Dirichlet boundary condition:

$$
\left\{\begin{array}{rll}
\Delta u_{1}=0 & & (x, y) \in \Omega \\
u_{1}=f & & (x, y) \in \partial \Omega
\end{array},\right.
$$

which can be solved efficiently by the ABIV method. The component $u_{2}$ takes care of the rest:

$$
\left\{\begin{aligned}
\Delta^{m} u_{2} & =0 & & (x, y) \in \Omega \\
u_{2} & =0 & (x, y) & \in \partial \Omega \\
\frac{\partial u_{2}}{\partial \nu} & =g:=\frac{\partial f}{\partial \nu}-\frac{\partial u_{1}}{\partial \nu} & & (x, y) \in \partial \Omega .
\end{aligned}\right.
$$

To find the component $u_{2}$ that satisfies the system (8) with certain degree of polyharmonicity $m$, we follow a strategy similar to that used in the ABIV method. The component $u_{2}$ shall be represented as a linear combination of a set of infinitely many polyharmonic functions. To this end, we find a sequence of functions denoted by $\left\{G_{k}\right\}_{k=1}^{\infty}$ which have the following properties:

1. $G_{k}=0 \quad(x, y) \in \partial \Omega$

2. $\frac{\partial G_{k}}{\partial \nu}=0 \quad(x, y) \in \partial \Omega \backslash\{(x, y) \mid x=1\}$

Note: such a property will allow one to treat four edges of the boundary separately.

3. $\left\{\left.\frac{\partial G_{k}}{\partial \nu}\right|_{x=1}\right\}_{k=1}^{\infty}$ is a basis of $L^{2}([0,1])$.

Moreover, define the function $h_{k}(x, y):=\sin (k \pi y) \frac{\sinh (k \pi x)}{\sinh (k \pi)}$, which is used in the ABIV Laplace solver with the following properties:

1. $\Delta h_{k}=0 \quad(x, y) \in \Omega$ 
2. $h_{k}=0 \quad(x, y) \in \partial \Omega \backslash\{(x, y) \mid x=1\}$

3. $\left\{\left.h_{k}\right|_{x=1}\right\}_{k=1}^{\infty}$ is a basis of $L^{2}([0,1])$.

A natural way to construct the functions $G_{k}$ is to seek a form, $G_{k}=h_{k}(x, y) p(x, y)$, where $p(x, y)$ is a polynomial. For the functions $G_{k}$ to satisfy the above three properties, we choose a polynomial $p$ such that it vanishes on the boundary $\partial \Omega$. The simplest choice is $p(x, y)=x(x-1) y(y-1)$. In fact, by direct computation one can show that the polyharmonicity of $G_{k}$ is equal to the summation of the polyharmonicity of $h_{k}$ and the degree of $p(x, y)$ (i.e., $\Delta^{(1+4)} G_{k}=0$ ). Hence, we conclude that the system (6) with $m=5$ is a polyharmonic system that can be computed fast and accurately using the existing ABIV method.

Once we find this polyharmonic $u$ component, the residual $v$ and its Fourier sine series expansion are computed as usual. We shall refer to this new version of PHLST as PHLST5.

In PHLST5 the Fourier sine coefficients of the residual $v$ have the same decaying rate as that of LLST, but the $\ell^{2}$ norm of the residual of PHLST5 is smaller for smooth images (see numerical experiments in Section 3). However, to satisfy the boundary conditions (5), there are plenty of other methods. In fact, we will show that it is possible to use scattered data interpolation methods such as a radial basis function to compute the $u$ component in our numerical experiment. However, it is computationally more expensive.

\subsection{An Algorithm to Compute PHLST5}

Because the system (7) can be solved directly by the ABIV method, our main task is to find a polyharmonic function that satisfies the system (8). We propose the following algorithm for this task. 
Step 1: Decompose (8) with $m=5$ into four independent subproblems.

$$
\begin{aligned}
& \left\{\begin{array}{rlrl}
\Delta^{5} u_{2}^{(1)} & =0 & & (x, y) \in \Omega \\
u_{2}^{(1)} & =0 & & (x, y) \in \partial \Omega \\
\frac{\partial u_{2}^{(1)}}{\partial \nu} & =g^{(1)} & & (x, y) \in \partial \Omega
\end{array} \quad g^{(1)}=\left\{\begin{array}{rl}
g & x \in(0,1), y=0 \\
0 & x \in(0,1), y=1 \\
0 & x=0, y \in(0,1) \\
0 & x=1, y \in(0,1)
\end{array},\right.\right. \\
& \left\{\begin{array}{rlrl}
\Delta^{5} u_{2}^{(2)} & =0 & & (x, y) \in \Omega \\
u_{2}^{(2)} & =0 & & (x, y) \in \partial \Omega \\
\frac{\partial u_{2}^{(2)}}{\partial \nu} & =g^{(2)} & & (x, y) \in \partial \Omega
\end{array} \quad g^{(2)}=\left\{\begin{array}{ll}
0 & x \in(0,1), y=0 \\
0 & x \in(0,1), y=1 \\
g & x=0, y \in(0,1) \\
0 & x=1, y \in(0,1)
\end{array},\right.\right.
\end{aligned}
$$

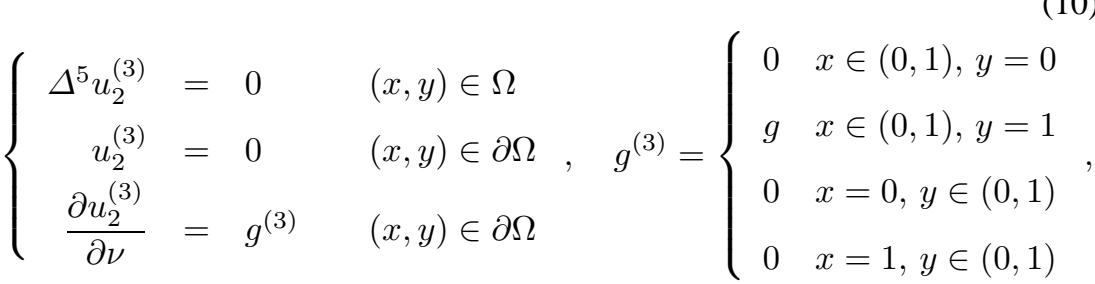

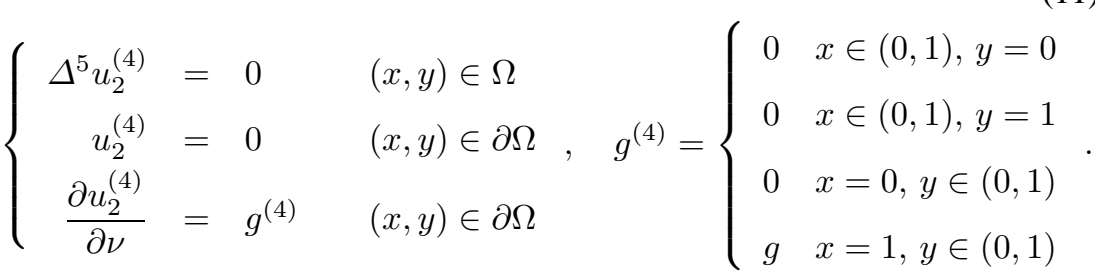

Step 2: Construct four sets of polyharmonic functions satisfying (9)-(12) respectively as follows:

$$
\begin{aligned}
& G_{1}:=\left\{G_{1 k}\right\}_{k=1}^{\infty}:=\left\{x(x-1) \sin (k \pi x) \cdot y(y-1) \frac{\sinh (k \pi(1-y))}{\sinh (k \pi)}\right\}_{k=1}^{\infty} \\
& G_{2}:=\left\{G_{2 k}\right\}_{k=1}^{\infty}:=\left\{x(x-1) \frac{\sinh (k \pi(1-x))}{\sinh (k \pi)} \cdot y(y-1) \sin (k \pi y)\right\}_{k=1}^{\infty} \\
& G_{3}:=\left\{G_{3 k}\right\}_{k=1}^{\infty}:=\left\{x(x-1) \sin (k \pi x) \cdot y(y-1) \frac{\sinh (k \pi y)}{\sinh (k \pi)}\right\}_{k=1}^{\infty} \\
& G_{4}:=\left\{G_{4 k}\right\}_{k=1}^{\infty}:=\left\{x(x-1) \frac{\sinh (k \pi x)}{\sinh (k \pi)} \cdot y(y-1) \sin (k \pi y)\right\}_{k=1}^{\infty}
\end{aligned}
$$

One can verify that each function in $G_{i}$ satisfies the zero Dirichlet boundary 
condition as required in (8) and its normal derivatives are zeros on three edges except the one with $g^{(i)}$ defined as in the equations (9)-(12).

Step 3: Compute the normal derivatives of the functions in each set $G_{i}$ on the boundary.

$$
\begin{aligned}
& P_{1}:=\{x(x-1) \sin (k \pi x)\}_{k=1}^{\infty}:=\left\{P_{1 k}\right\}_{k=1}^{\infty} x \in(0,1), y=0 \\
& P_{2}:=\{y(y-1) \sin (k \pi y)\}_{k=1}^{\infty}:=\left\{P_{2 k}\right\}_{k=1}^{\infty} \quad x=0, y \in(0,1) \\
& P_{3}:=\{x(x-1) \sin (k \pi x)\}_{k=1}^{\infty}:=\left\{P_{3 k}\right\}_{k=1}^{\infty} \quad x \in(0,1), y=1 \\
& P_{4}:=\{y(y-1) \sin (k \pi y)\}_{k=1}^{\infty}:=\left\{P_{4 k}\right\}_{k=1}^{\infty} \quad x=1, y \in(0,1),
\end{aligned}
$$

where $P_{i k}$ is the normal derivative of $G_{i k}$ on an appropriate boundary depending on the values of $i$.

Step 4: Expand $g^{(i)}=\sum_{k=1}^{\infty} w_{k}^{(i)} P_{i k}, i=1, \ldots, 4$, where $w_{k}^{(i)}, k \in \mathbb{N}$ are real coefficients to be identified through this expansion.

Step 5: Set $u_{2}=\sum_{i=1}^{4} \sum_{k=1}^{\infty} w_{k}^{(i)} G_{i k}$. This is a desired polyharmonic function with the degree of polyharmonicity $m=5$.

In practice, if we discretize the image $f$ at $\left(x_{i}, y_{j}\right)=(i / N, j / N), i, j=0,1, \ldots, N-$ $1, N$, and view it as a matrix of size $(N+1) \times(N+1)$, then in Steps $2-4$ above, one should discard the first and last entries on the boundary and use $N-1$ terms instead of infinitely many terms. The overall computational cost is about twice as that of the ABIV method for solving Laplace's equation if we do not count the cost for estimating normal derivatives. We remark that in Step 4, we first divide the boundary functions $g^{(i)}$ on $(0,1)$ by the quadratic polynomial $x(x-1)$ for $i=1,3$ and $y(y-1)$ for $i=2,4$. Since the two endpoints of the interval are not included in the division, there is no numerical blowup there. Then, we expand the results into Fourier sine series. Since $u_{2}=0$ on the four boundary sides, one can easily see that $g^{(i)}$ are zeros at the endpoints of each side, so that these functions are still suitable functions to be expanded into Fourier sine series even after divided by $x(x-1)$ or $y(y-1)$. 
The algorithm by itself is now complete. We still need to decide how to compute the normal derivative $g$ on the boundary in the equations (9)-(12). If one takes a close look at the ABIV Laplace solver, the solution $u_{1}$ of (7) is given in an analytic form as shown in Appendix A. We simply compute the normal derivatives of the $u_{1}$ component from two sides of the boundary (between two blocks) by using its analytic form, and take their averages. Those values will be fed to the algorithm as our estimated normal derivatives $\frac{\partial f}{\partial \nu}$.

We summarize some features of PHLST5 below:

- Since the estimation of the values of the normal derivative is computed from the $u_{1}$ component, there is no need to store them. This implies that PHLST5 only requires the same storage and information as LLST does.

- The computational cost of the $u\left(=u_{1}+u_{2}\right)$ component in PHLST5 is just twice as much as that of LLST.

- Since additional information of the first order normal derivatives are matched on the grids, PHLST5 produces a globally smoother $u$ component than LLST.

\section{Numerical Experiments}

In this section, we shall report the results of our numerical experiments and compare the performance of PHLST5 with that of LLST and that of the method using scattered data approximation by radial basis functions.

\subsection{Experiments with Synthetic Data}

We set the domain $\bar{\Omega}=[0,1]^{2}$. The first image we have chosen is a smooth and flat function $f$ defined by

$$
f(x, y)=\sin (x+2 y) \mathrm{e}^{-3\left((x-0.2)^{2}+(y-0.4)^{2}\right)} .
$$


We sample the image on the regular lattice with $129 \times 129$ grid points. Note that the values of the first order normal derivatives on the boundary used in PHLST5 can be calculated analytically. Figure 1 shows the residuals of LLST and PHLST5 in the spatial domain. PHLST5 clearly outperforms LLST in terms of the size of the residual $v$. In fact, the residual component $v$ of PHLST5 has much smaller $\ell^{2}$ norm than that of LLST as we can see from Figure 1 (b) and (c). The ratio of $\|v\|_{2}$ to $\|f\|_{2}$ is 0.1317 in PHLST5 while that of LLST is 0.4969 . This implies that the polyharmonic $u$ component in PHLST5 predicts and approximates the original $f$ better than that in LLST in terms of the $\ell^{2}$ norm.

Next, we examine a more oscillatory image,

$$
f(x, y)=\sin (20(x+2 y)) \mathrm{e}^{-3\left((x-0.2)^{2}+(y-0.4)^{2}\right)} .
$$

The image is sampled on a regular lattice with $1025 \times 1025$ grid points. The PHLST5 algorithm is applied at different levels of segmentation this time. Denote by $J$ the level of dyadic splitting ( $J=0$ means no splitting). We shall refer to the union of polyharmonic components and the union of residual components of all segmented pieces as $u$ and $v$ respectively. In this experiment we do not use the analytic derivative information from the given formula of the image $f$. Rather, we apply the estimation method described at the end of Section 2.3. The relative $\ell^{2}$ norm (i.e., $\frac{\|v\|}{\|f\|}$ ) is computed as the error (abbreviated as Err in Figures 2 and 3). One can see that in the coarser levels $(J=0,1)$, the $v$ component (i.e., the union of the local residual pieces) of LLST is smaller than that of PHLST5. However as we further segment the image into local pieces, PHLST5 outperforms LLST remarkably. This is because that PHLST5 includes more information from the boundary. Consequently it predicts the original function better. Figures 2 and 3 compare the convergence of $u$ components to our original function as the level of splitting gets deeper. 


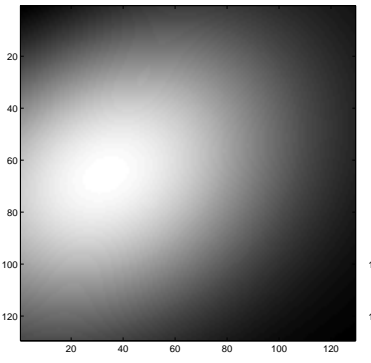

(a) Original

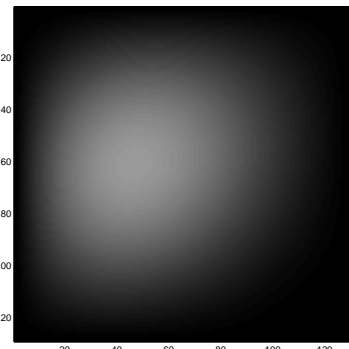

(b) LLST residual

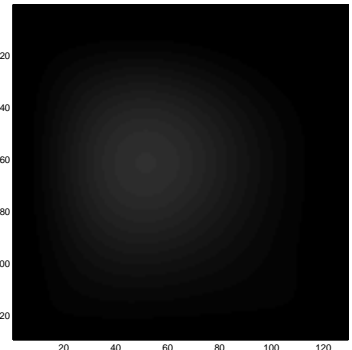

(c) PHLST5 residual

Figure 1: Comparison of the residuals of LLST and PHLST5 using smooth data.

\subsection{Experiments with Real Images}

We now report our numerical experiments using real images. We have selected two regions of the popular "Barbara" image for our experiments. One is around the face area (smooth region) with the scarf (some oscillations). The other one is around the leg area (very oscillatory). The size of both images are $129 \times 129$ pixels. We first compute the $u$, the union of the local polyharmonic components $\left(u_{i}\right.$ 's), and expand the $v$, the union of the local residual components $\left(v_{i}\right.$ 's), into a Fourier sine series. Then we approximate the residual $v$ with a few large Fourier sine coefficients and reconstruct the original image via the computed polyharmonic component $u$ and the approximated residual $v$. The approximation qualities were then compared in PSNR (i.e., Peak Signal-to-Noise Ratio) and MSSIM (Multiscale Structure SIMilarity) [22]. MSSIM is an image similarity index in the interval $[0,1]$ that compares the difference between two images in terms of luminance, contrast, and structure. The closer MSSIM to 1, the more similar the compared two images are. The values of the first order normal derivative are computed using the method described in Section 2.3 again. In addition, we also compare the results of LLST and PHLST5 with the component $u$ computed from the radial basis function transform (RDT) $[9,10,11,17,6,20]$. In RDT, we choose the most often applied radial basis function-a multiquadric $\phi(\boldsymbol{x})=\sqrt{\|\boldsymbol{x}\|_{2}^{2}+1}$. The component $u$ 

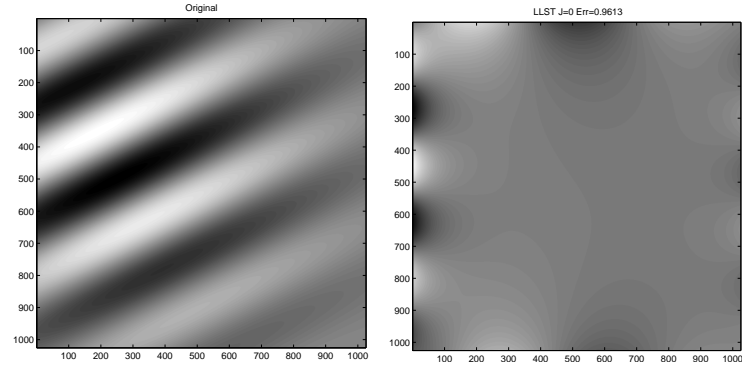

(b) $J=0$, Err $=0.9613$

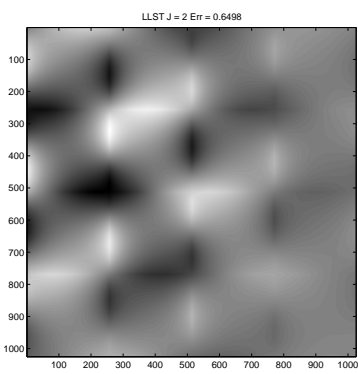

(d) $J=2$, Err $=0.6498$

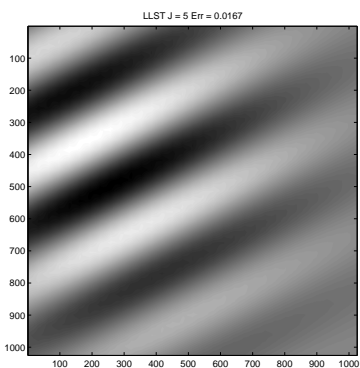

(g) $J=5$, Err $=0.0167$
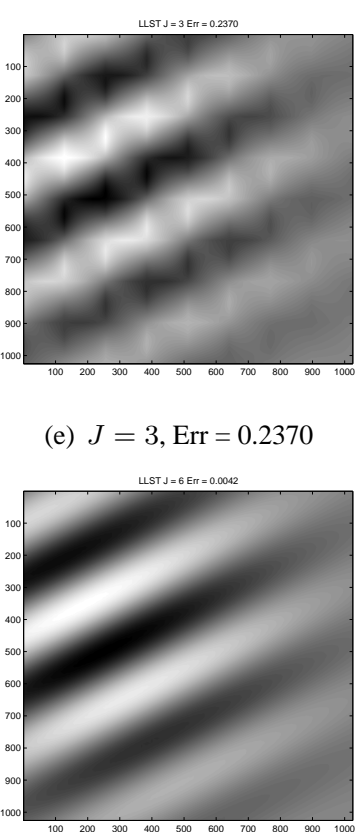

(h) $J=6$, Err $=0.0042$ (e) $J=3$, Err $=0.2370$

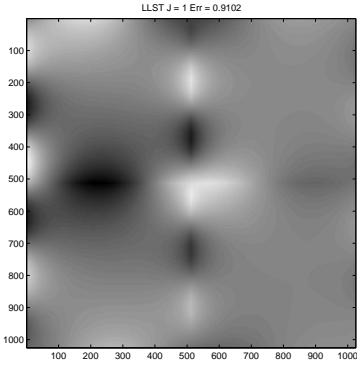

(c) $J=1$, Err $=0.9102$

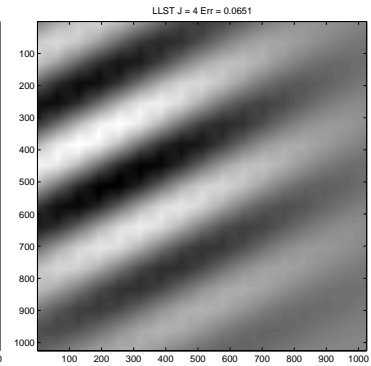

(f) $J=4$, Err $=0.0651$

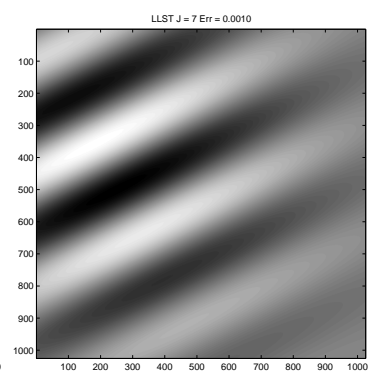

(i) $J=7$, Err $=0.0010$

Figure 2: The $u$ component of LLST in different levels of splitting. 

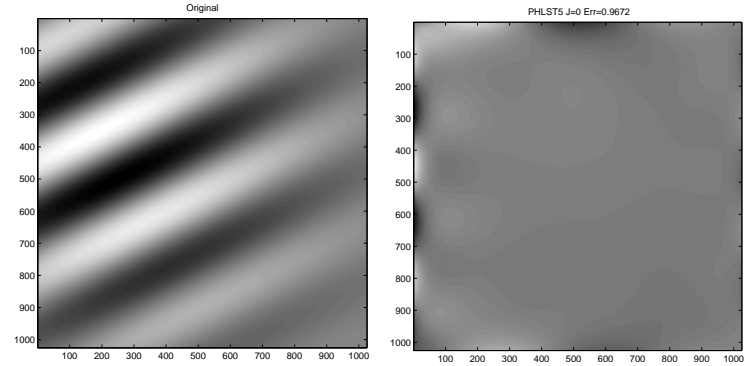

(b) $J=0$, Err $=0.9672$

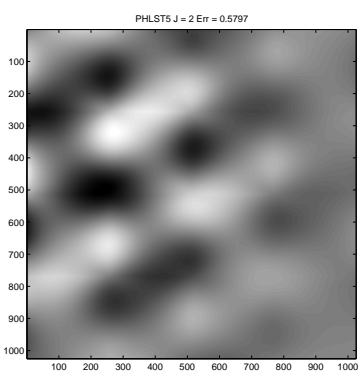

(d) $J=2$, Err $=0.5797$

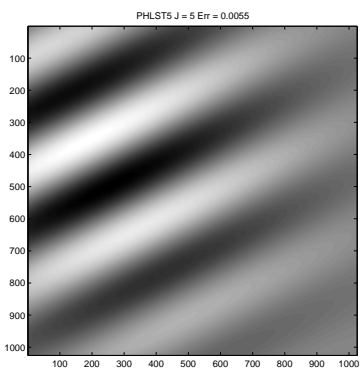

(g) $J=5$, Err $=0.0055$

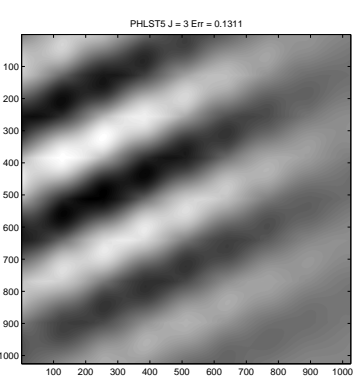

(e) $J=3$, Err $=0.1311$

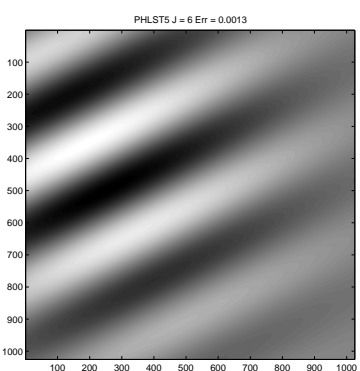

(h) $J=6$, Err $=0.0013$

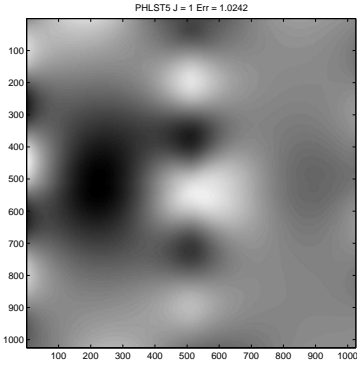

(c) $J=1$, Err $=1.0242$

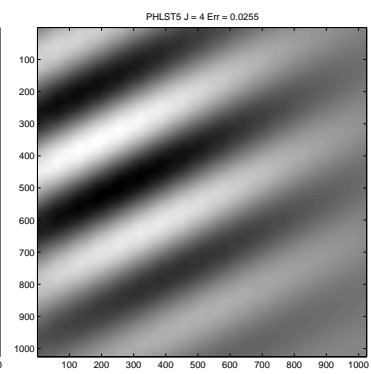

(f) $J=4$, Err $=0.0255$

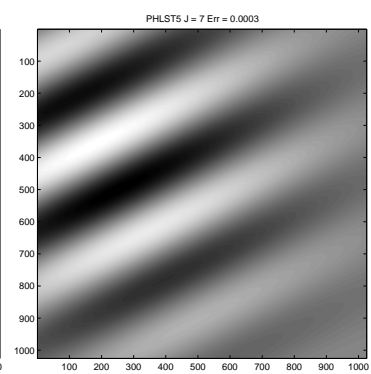

(i) $J=7$, Err $=0.0003$

Figure 3: The $u$ component of PHLST5 in different levels of splitting. 
is calculated by the standard radial function approximant

$$
u(\boldsymbol{x})=\sum_{\boldsymbol{y}_{i} \in S} c_{i} \phi\left(\boldsymbol{x}-\boldsymbol{y}_{i}\right),
$$

where $S$ is the set of grid points ("centers" in radial basis function jargon) where we sample our image data. The coefficients $c_{i}$ are found by solving the linear system below resulted from the collocation

$$
\sum_{\boldsymbol{y}_{i} \in S} c_{i} \phi\left(\boldsymbol{x}_{j}-\boldsymbol{y}_{i}\right)=f\left(\boldsymbol{x}_{j}\right), \quad \boldsymbol{x}_{j} \in S,
$$

where $f\left(\boldsymbol{x}_{j}\right)$ is the gray level value of the image at the given spot $\boldsymbol{x}_{j}$. We note that the component $u$ computed by RDT is a function in $C^{\infty}$. In this experiment, we split the image domain homogeneously into $4 \times 4$ blocks (i.e., $32 \times 32$ pixels within each block) and $8 \times 8$ blocks (i.e., $16 \times 16$ pixels within each block). Figures 4 and 5 show the $u$ components from RDT, LLST and PHLST5 of the face image and leg image respectively. Figure 6 shows the quality difference measured by PSNR and MSSIM. Figure 7 shows the zoomed up version of Figure 6 of the face part of the Barbara image. Similarly Figures 8 and 9 are for the leg part of the Barbara image.

From these figures, we observe the following:

- In the face image (smooth):

1. The $u$ component of PHLST5 shows a visual improvement over that of LLST in the eye areas.

2. RDT performs the best, followed by PHLST5 and then LLST.

3. With further splitting the image domain, the measurement curves (PSNR, MSSIM) of RDT and PHLST5 are separated from that of LLST even more.

- In the leg image (textured):

1. RDT shows a significant advantage in terms of PSNR over LLST and PHLST5. But three approaches are not significantly different in terms of MSSIM. 
2. Further splitting the image domain benefits RDT and PHLST5 in terms of PSNR.

One can see that RDT outperforms the other two methods in both regions in terms of PSNR. This is because RDT is a globally smooth interpolation. In addition to matching the boundary values of a single block, it matches the boundary values of all the blocks at once. On the other hand, each $u$ component in PHLST5 is local: it uses the boundary values of each block and the averages of the derivatives among the neighbouring blocks.

In order to see the visual quality of these approximations, we further examine the reconstruction quality of this Barbara face image. Figures 10-15 show the reconstructions and the errors by using the top 1500 coefficients (i.e. $10.41 \%$ of the total coefficients of the residual $v$ ) with $8 \times 8$ segmentation of the $129 \times 129$ Barbara face image. From these results it is clear that the quality of PHLST5 approximation is better than that of LLST approximation. The features from eyes, nose and mouth areas are more obviously shown in the reconstruction error of LLST. In addition, blocking artifacts present in LLST are less noticeable in PHLST5.

\section{Discussion}

We have described a new, practical, and improved version of PHLST called PHLST5 that uses a 5 th degree polyharmonic function as the ployharmonic component $u$. The values of the polyharmonic component and of the first order normal derivative match those of the original function $f$ on the boundary. The Fourier sine coefficients of the residual $v=f-u$ have the same decaying rate but much smaller energy compared to those of LLST. We have demonstrated the advantage of PHLST5 over LLST using two synthetic images in terms of the size of the residual. Our experiments on the real images confirm that PHLST5 beats LLST at smooth regions. Similarly to the ABIV method, our algorithm to compute the PHLST5 representation of an input image is fast, accu- 

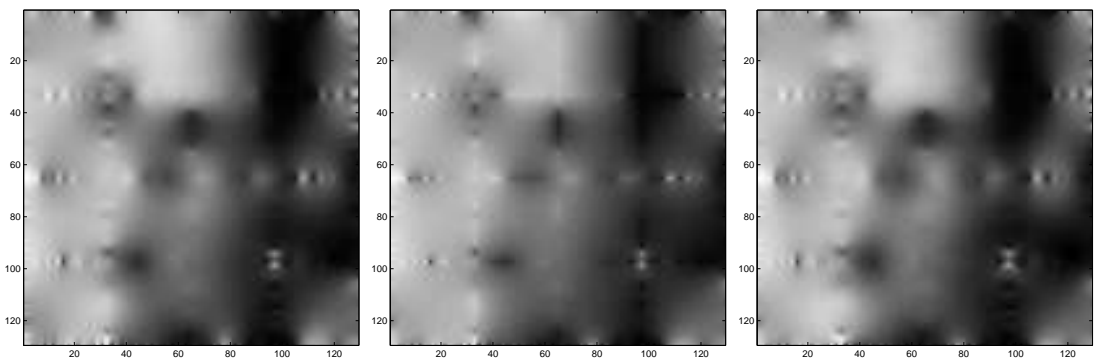

(a) The $u$ component of RDT (b) The $u$ component of LLST (c) The $u$ component of

$(4 \times 4$ segmentation $)$

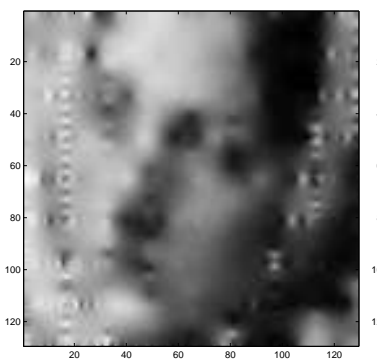

(d) The $u$ component of RDT
$(4 \times 4$ segmentation $)$

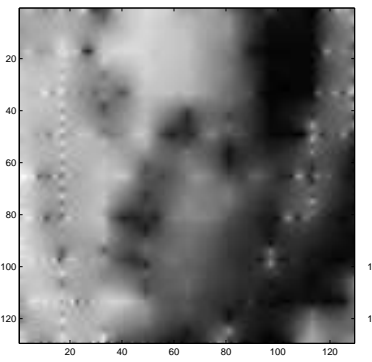

PHLST5 $(4 \times 4$ segmentation $)$

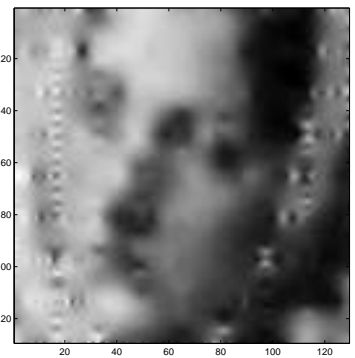

(e) The $u$ component of LLST (f)

$(8 \times 8$ segmentation $)$
PHLST5 $(8 \times 8$ segmentation $)$

Figure 4: $u$ components from RDT, LLST and PHLST5 in the face area. 

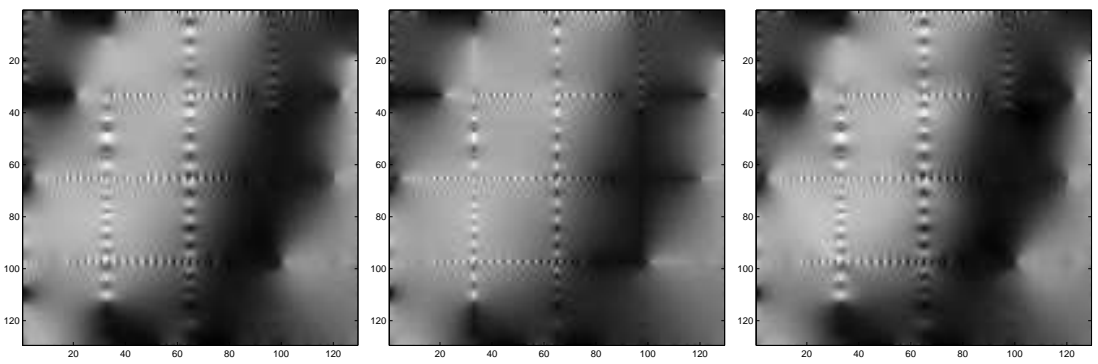

(a) The $u$ component of RDT (b) The $u$ component of LLST (c) The $u$ component of $(4 \times 4$ segmentation $)$ $(4 \times 4$ segmentation $)$ PHLST5 $(4 \times 4$ segmentation $)$
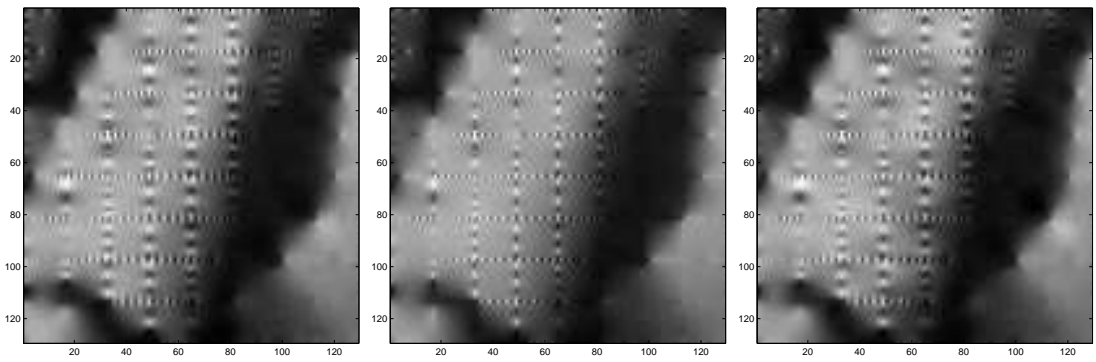

(d) The $u$ component of RDT (e) The $u$ component of LLST (f) The $u$ component of $(8 \times 8$ segmentation $)$ $(8 \times 8$ segmentation $)$ PHLST5 $(8 \times 8$ segmentation $)$

Figure 5: $u$ components from RDT, LLST and PHLST5 in the leg area. 

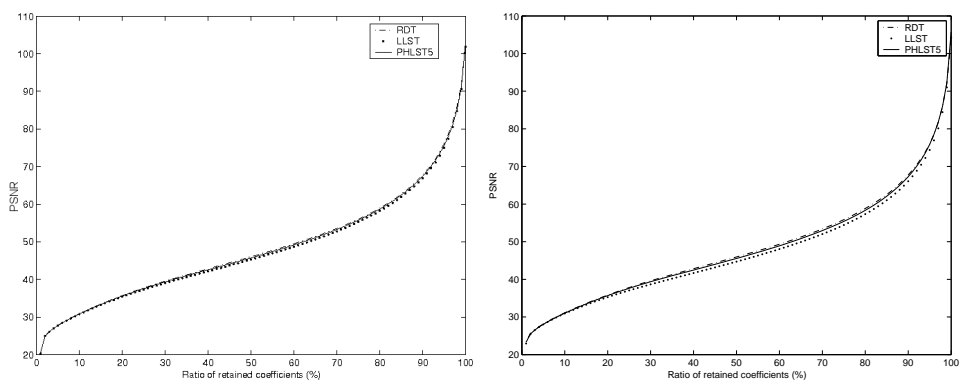

(a) PSNR $4 \times 4$ segmentation in Face Im- (b) PSNR $8 \times 8$ segmentation in Face Image Area age Area
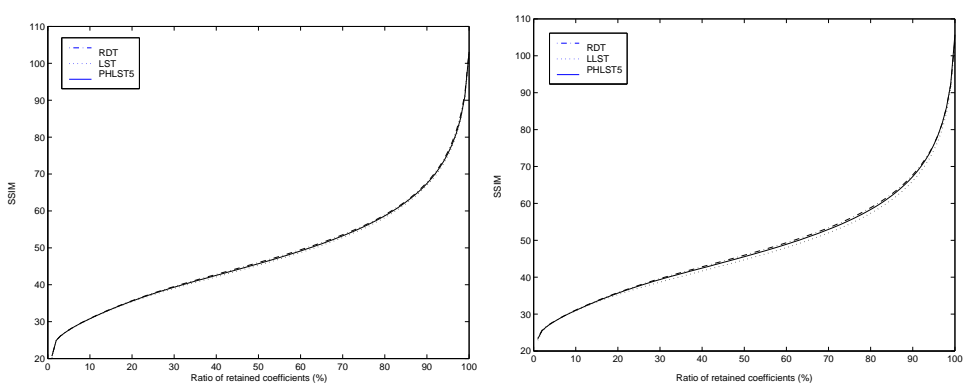

(c) MSSIM $4 \times 4$ segmentation in Face Im- (d) MSSIM $8 \times 8$ segmentation in Face Image Area age Area

Figure 6: Quality measurements of face image area. 

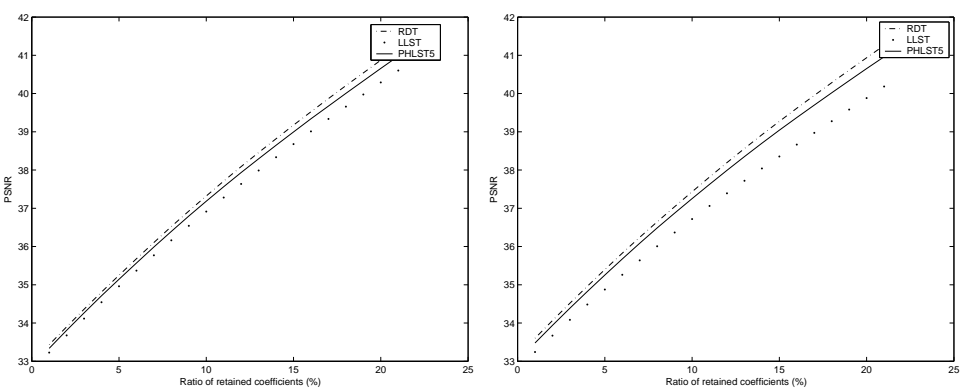

(a) PSNR $4 \times 4$ segmentation in Face Im- (b) PSNR $8 \times 8$ segmentation in Face Image Area age Area
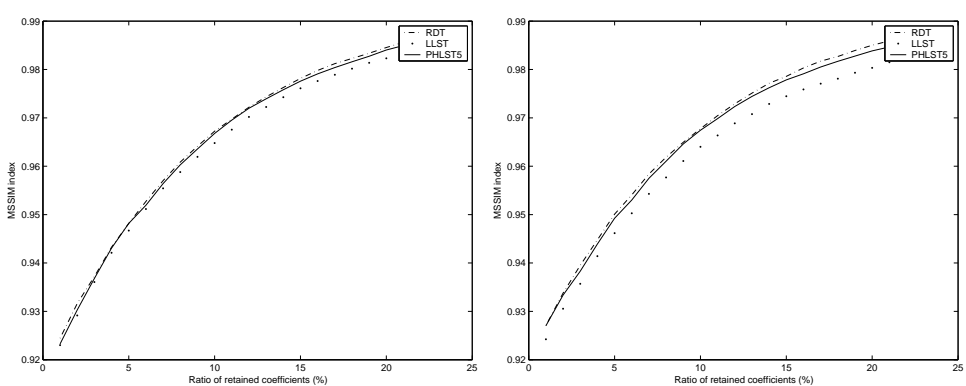

(c) MSSIM $4 \times 4$ segmentation in Face Im- (d) MSSIM $8 \times 8$ segmentation in Face Image Area age Area

Figure 7: Zoom up version of Figure 6. 

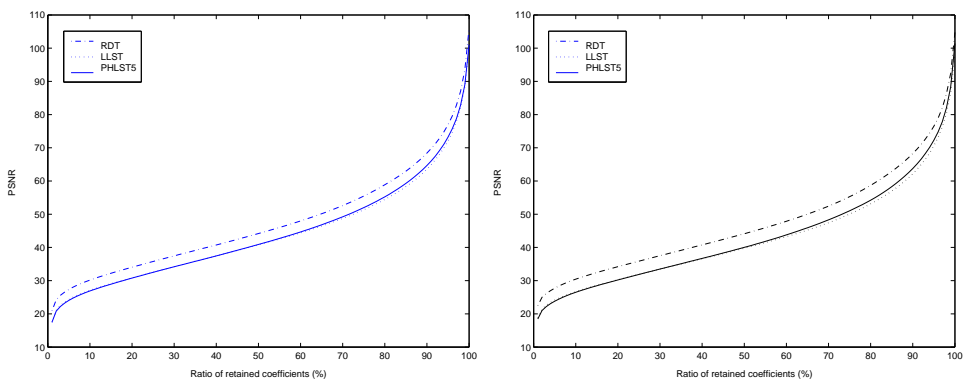

(a) PSNR $4 \times 4$ segmentation in Leg Image (b) PSNR $8 \times 8$ segmentation in Leg Image

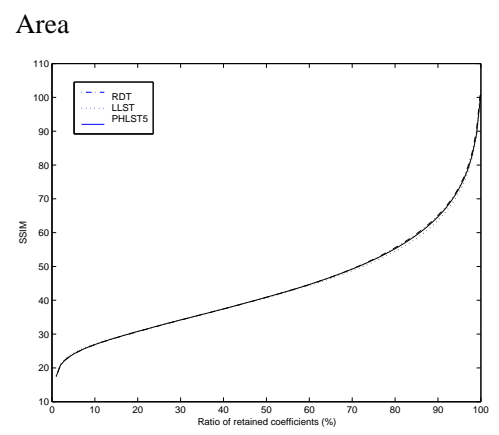

Area

(c) MSSIM $4 \times 4$ segmentation in Leg Im- (d) MSSIM $8 \times 8$ segmentation in Leg Image Area age Area

Figure 8: Quality measurements of leg image area. 

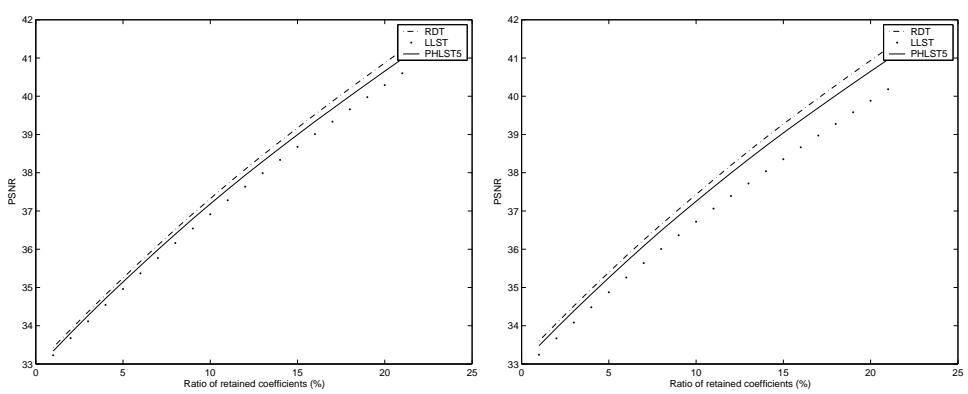

(a) PSNR $4 \times 4$ segmentation in Leg Image (b) PSNR $8 \times 8$ segmentation in Leg Image

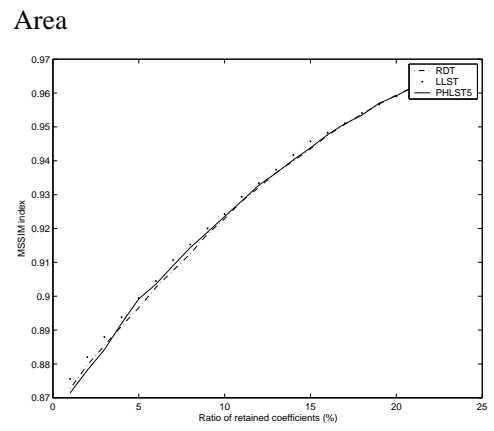

Area

(c) MSSIM $4 \times 4$ segmentation in Leg Im- (d) MSSIM $8 \times 8$ segmentation in Leg Image Area age Area

Figure 9: Zoom up version of Figure 8. 


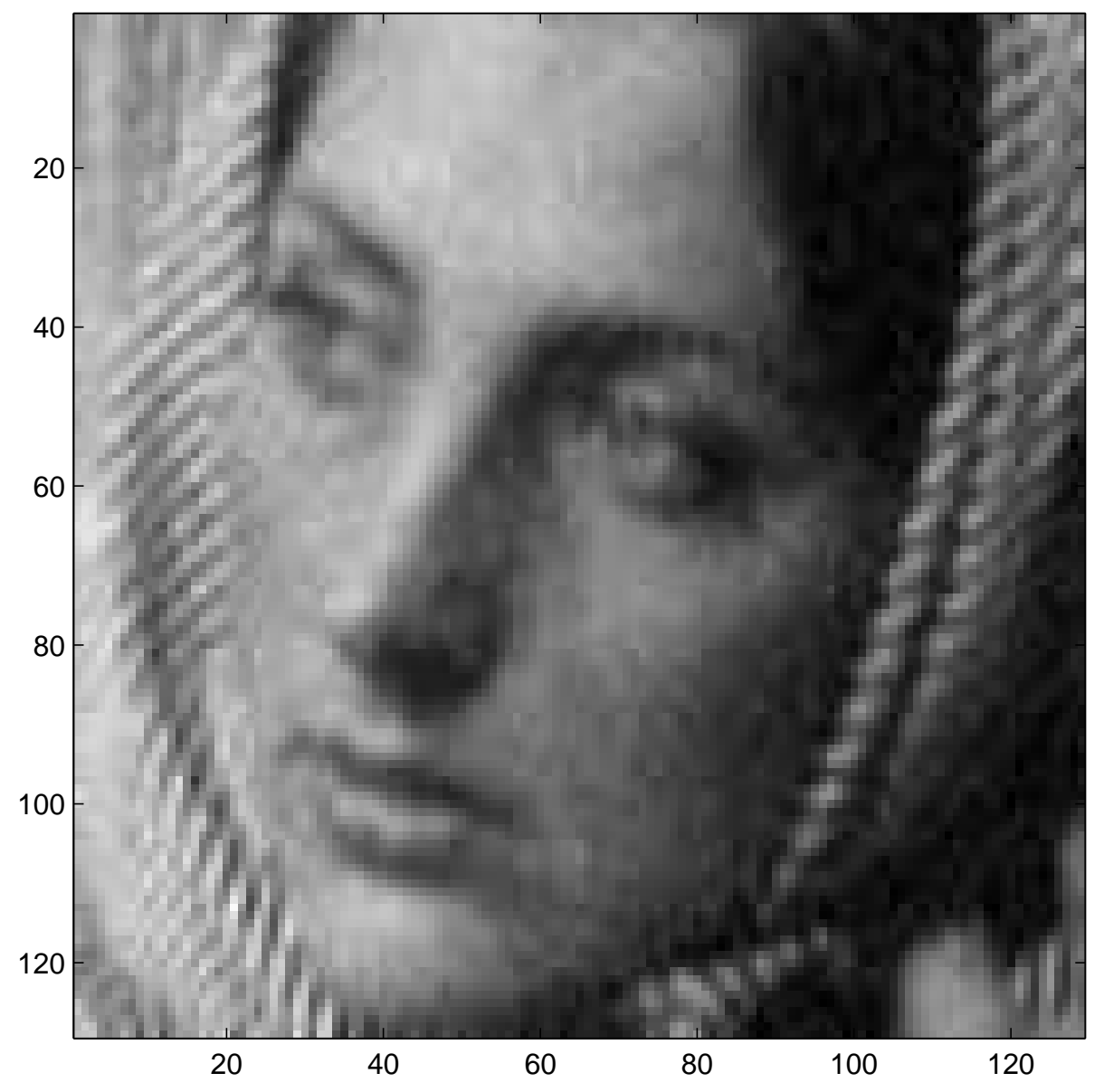

Figure 10: The 1500 coefficient reconstruction from RDT. 


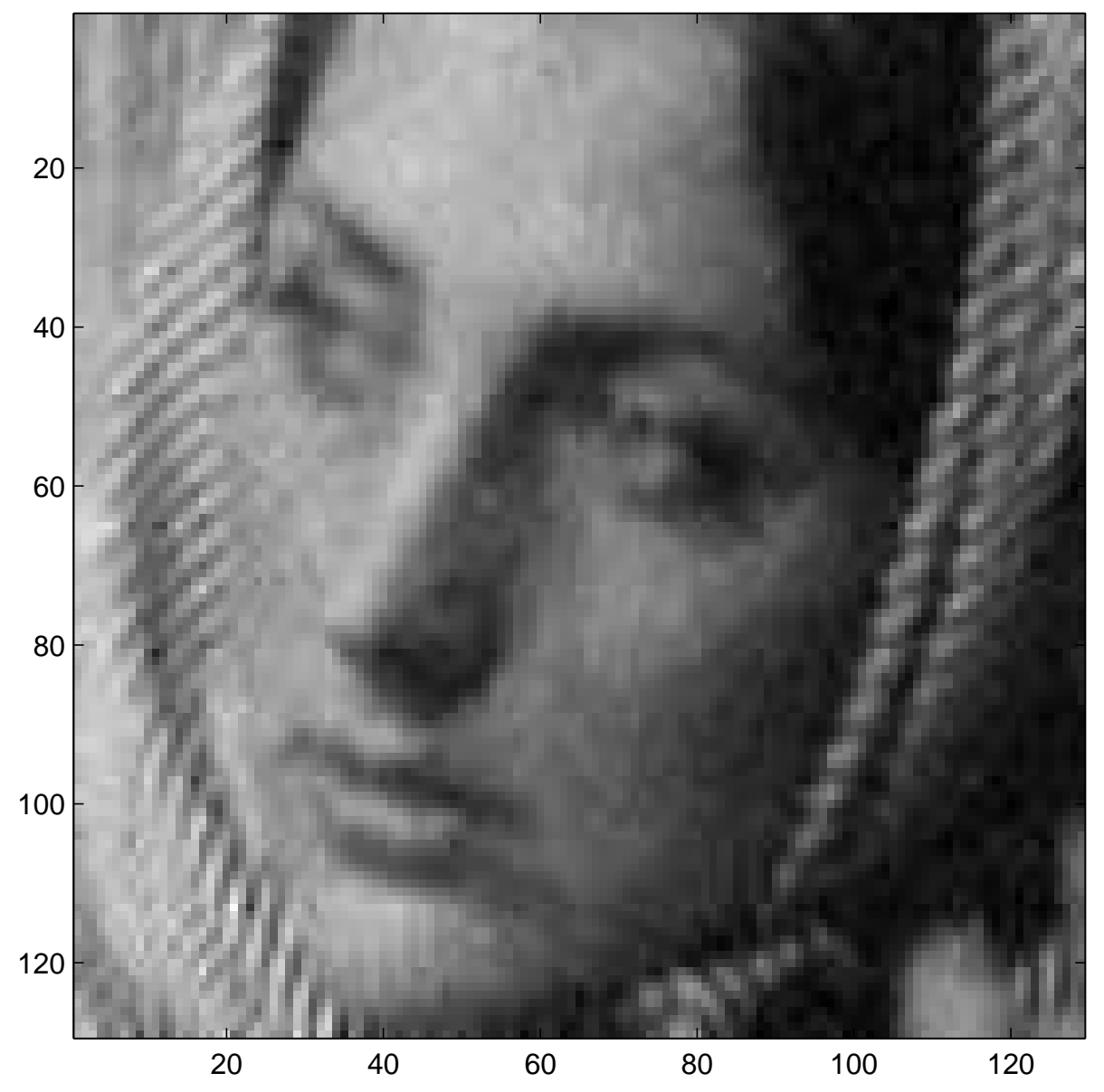

Figure 11: The 1500 coefficient reconstruction from LLST. 


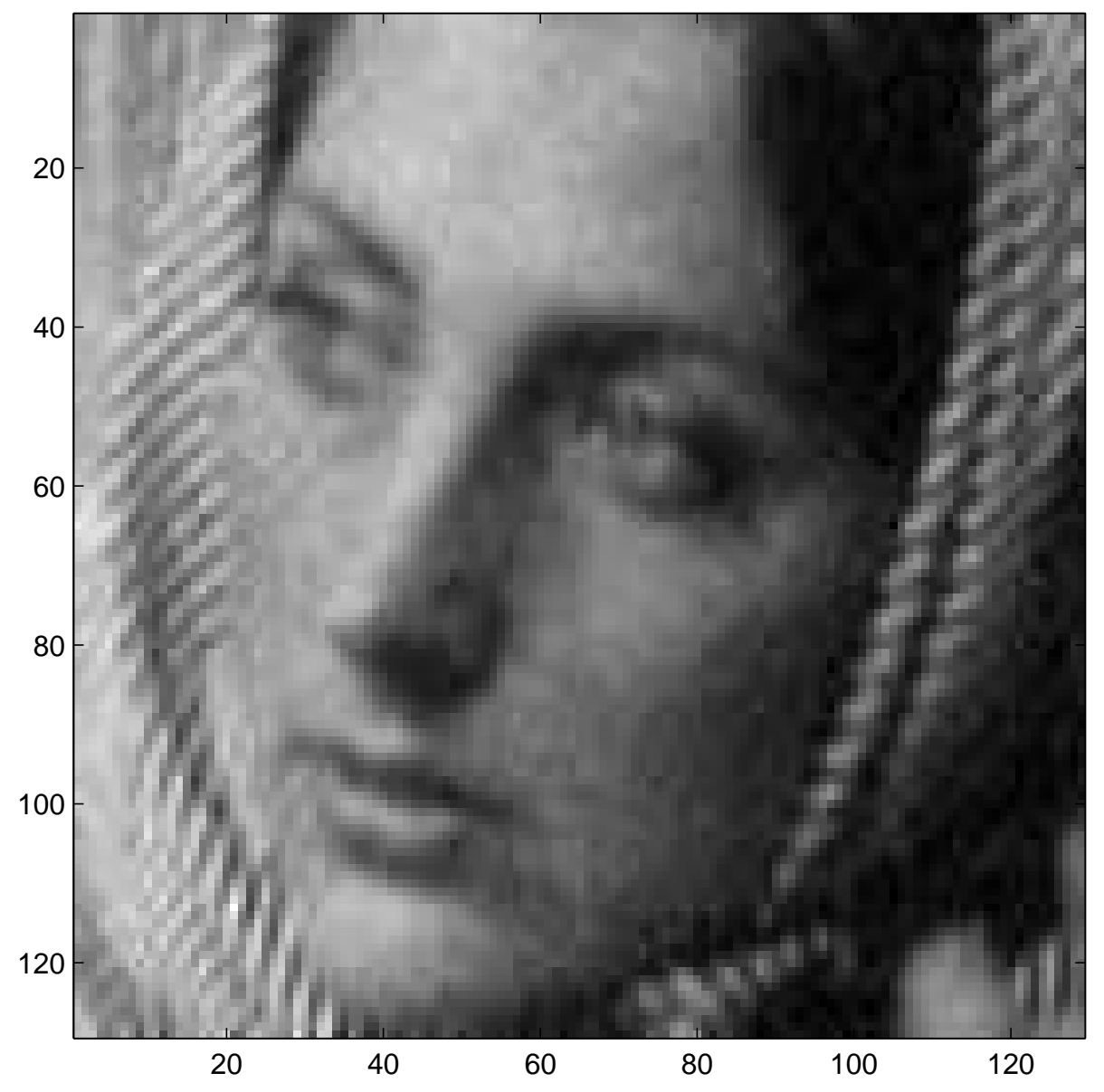

Figure 12: The 1500 coefficient reconstruction from PHLST5. 


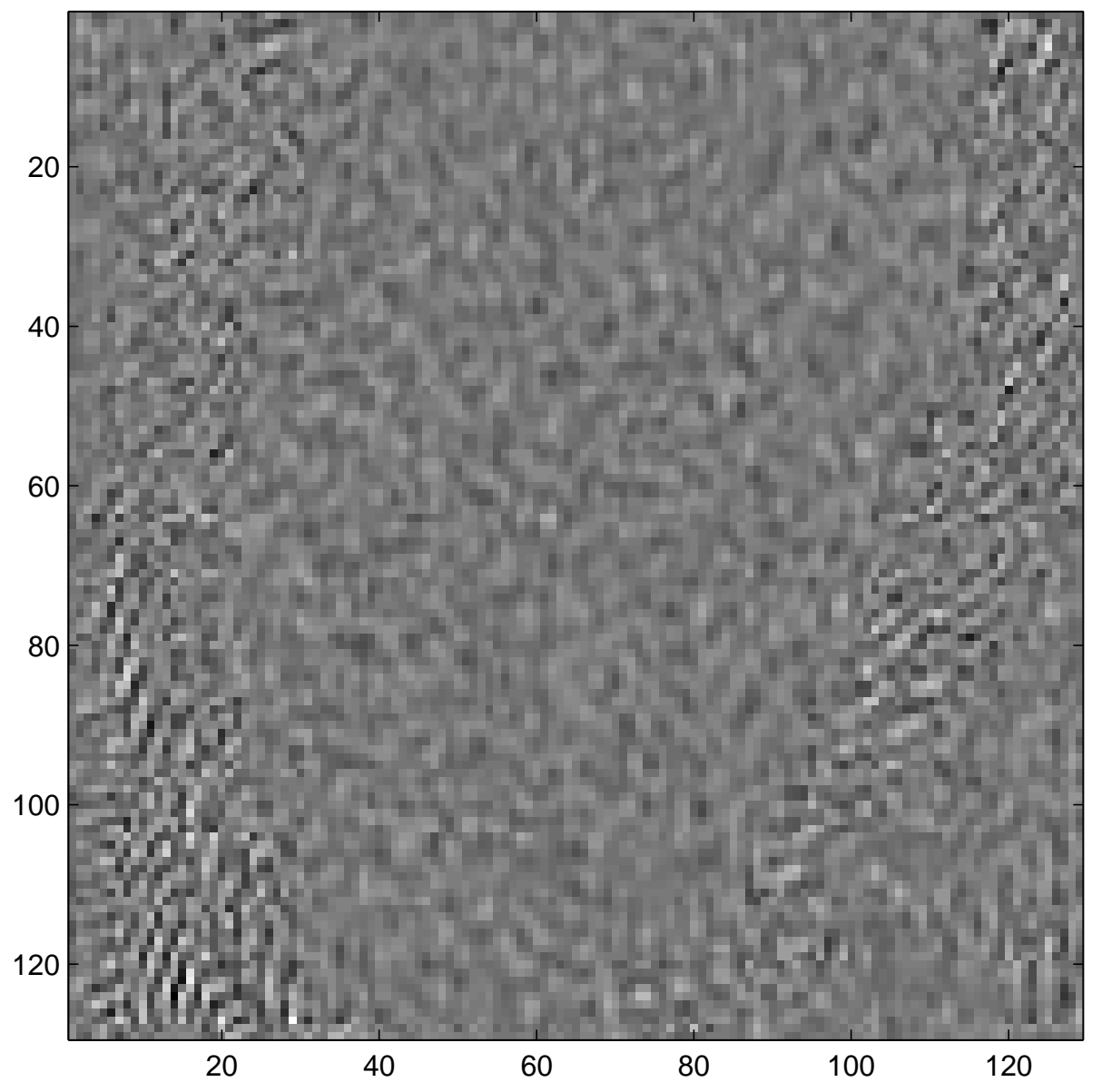

Figure 13: The error component from RDT. 


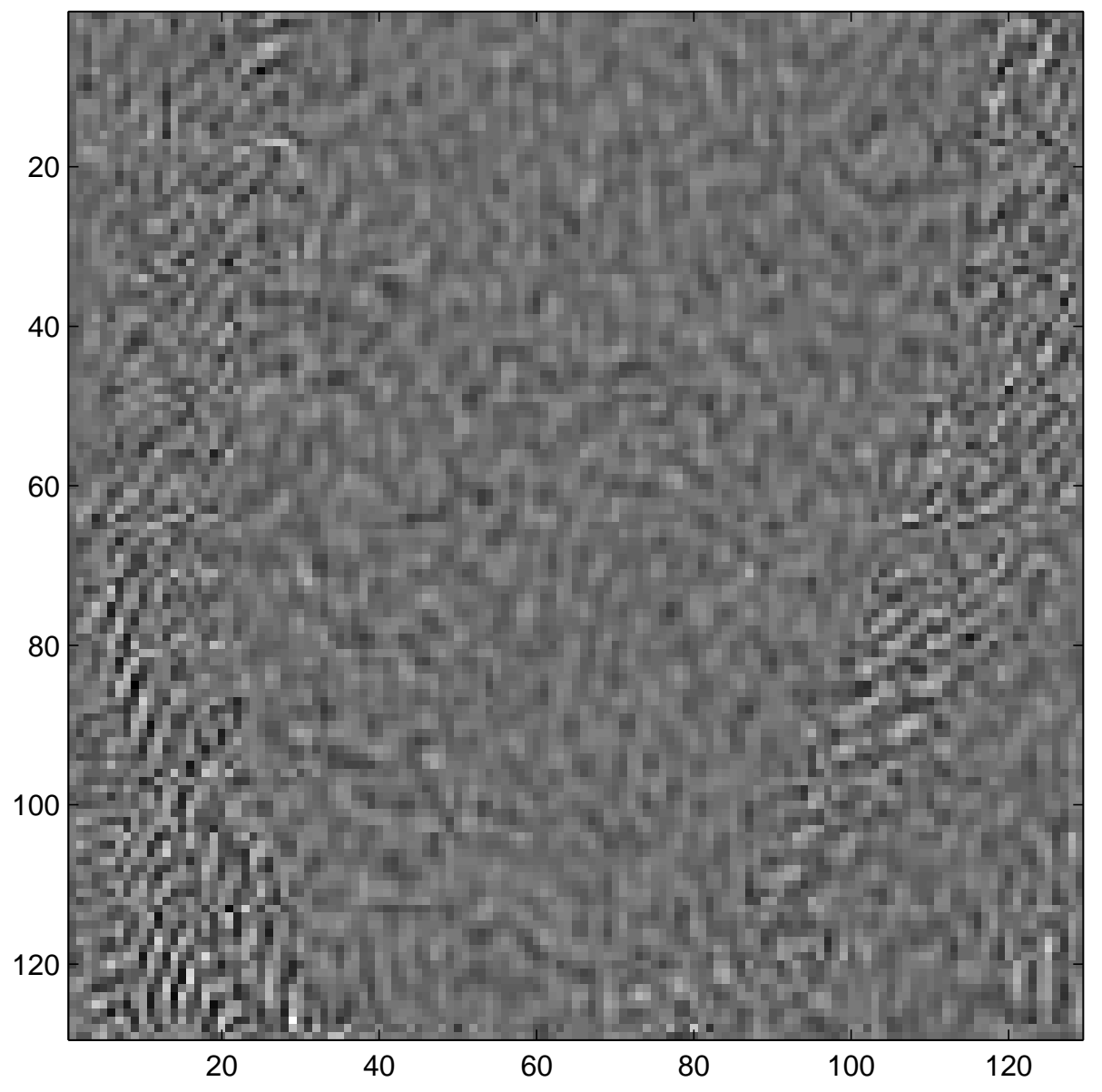

Figure 14: The error component from LLST. 


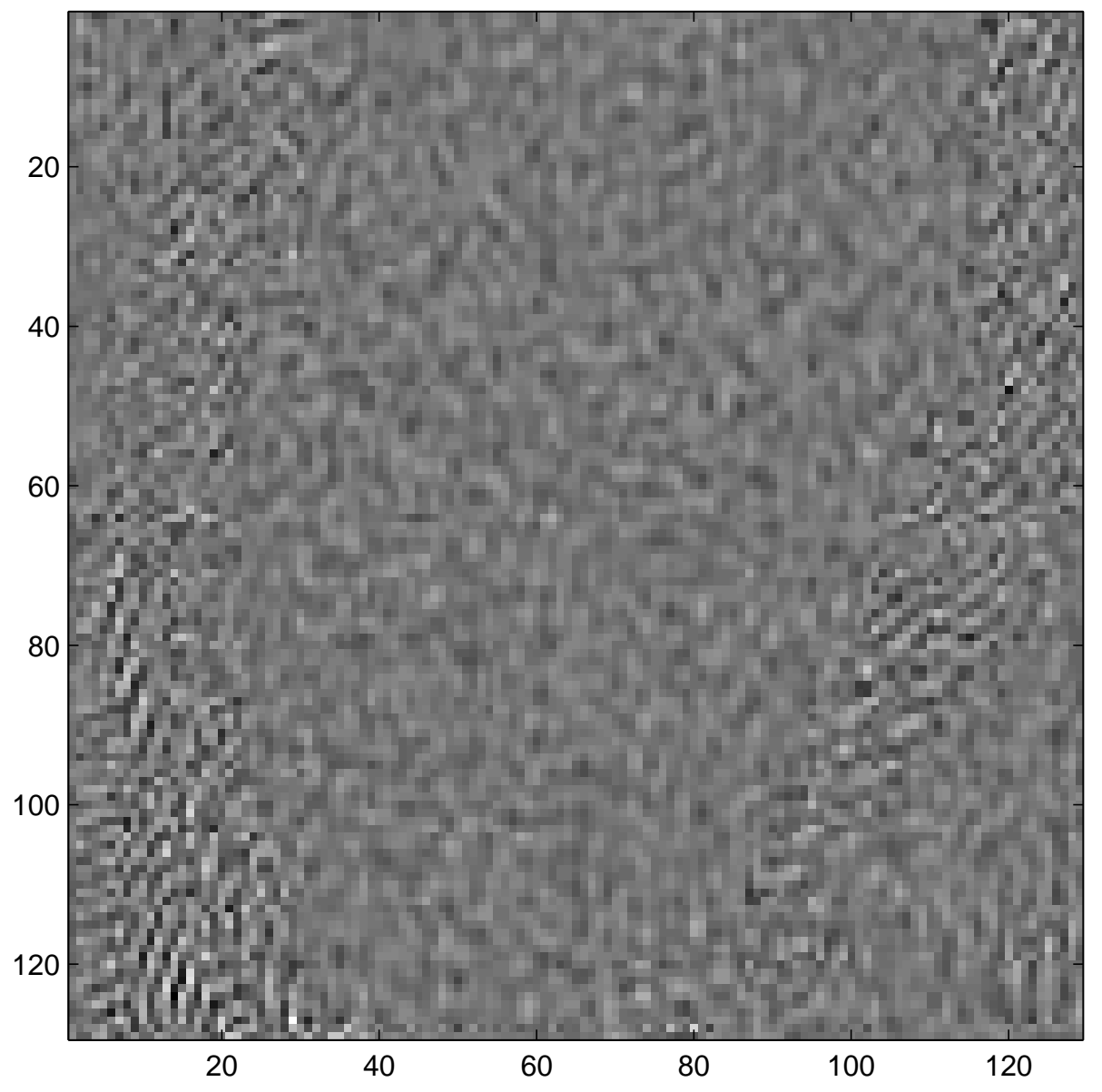

Figure 15: The error component from PHLST5. 
rate, and based on an analytic formula. Thus, this method can also be used for image approximation and zooming without suffering from the Gibbs phenomenon. However, the current PHLST5 algorithm only works with rectangular domains. This is due to our treatment of the boundary conditions (see Equations (9)-(12)). We also showed a radial basis function transform (RDT) to compute the $u$ component. It provides a globally smooth interpolation. Hence in smooth regions, it predicts the original image well and has a very small $v$ component. The computational cost of RDT, however, is huge since it requires solving a full linear system (15).

The PHLST5 can also be viewed as the following approximation problem. Let $U$ be the set of functions defined by

$$
U:=\left\{u \in C^{1}(\Omega): \Delta^{5}\left(\chi_{\Omega_{j}} u\right)=0, \bar{\Omega}=\cup_{j} \bar{\Omega}_{j}\right\} .
$$

In other words, the set $U$ consists of functions in $C^{1}(\Omega)$ subject to that their restrictions to each subdomain $\Omega_{j}$ are polyharmonic of degree 5 . One can see immediately that $U$ is a subspace of $C^{1}(\Omega)$. Now, given an image $f \in L^{2}(\Omega)$, let us decompose $f$ as $f=u+v$ where $u \in U$ is an approximation to $f$. The PHLST5 algorithm provides a way to find such $u$. It would be ideal if we could find the least squares approximation $u^{*} \in U$ to $f \in L^{2}(\Omega)$, i.e., $u^{*}=\arg \min _{u \in U}\|f-u\|_{L^{2}(\Omega)}$. Such $u^{*}$ would, of course, guarantee that the corresponding $v$ component, say $v^{*}$ is minimal in terms of energy. Moreover, even if the original image $f$ contains noise, the resulting decomposition $f=u^{*}+v^{*}$ still makes good sense. Although our PHLST5 algorithm provides a good and smooth approximation $u \in U$ to $f$, our $u$ is not the least squares solution $u^{*}$ unfortunately. Deriving a practical algorithm to compute such least squares solutions has the first priority in our research along this direction.

There are a few more issues still left open. First, the theoretical aspect of PHLST5 is still open. This is not an easy task since we are using the 5th degree polyharmonic operator that is not well studied. At this point we only numerically demonstrated the polyharmonic $u$ component of PHLST5 converges to the original function much faster than LLST if we further split the domain. We will set this topic also as our high priority 
research. Second, PHLST5 has been shown to improve LLST at smooth regions of an image. It is necessary to come up with a criterion while splitting an image so that we can tell a priori where exactly PHLST5 should be applied.

Finally, due to the difficulty of estimating higher order derivatives, we consider PHLST5 as the practical limitation of implementing PHLST with higher degree polyharmonicity.

\section{Acknowledgment}

This work was partially supported by the grants ONR YIP N00014-00-1-0469, ONR N00014-06-1-0615, ONR N00014-07-1-0166, and NSF DMS-0410406. The authors thank the anonymous reviewers for their comments and criticisms that significantly improved the quality of this paper.

\section{A The Laplace Solver of Averbuch, Braverman, Israeli \& Vozovoi}

If the underlying domain $\Omega$ and its subdomains $\Omega_{j}$ 's are rectangular regions in $\mathbb{R}^{d}$, which is the most practical case in numerical implementation, we can employ the Discrete Sine Transform (DST) based on the FFT algorithm to rapidly compute both the polyharmonic components and the Fourier sine series expansions of the residual components. In particular, we use the algorithm proposed by Averbuch, Braverman, Israeli, and Vozovoi [1, 4], which seems to us the most natural and practical Laplace/Poisson equation solver on rectangular domains. The ABIV method offers more accurate solutions than those based on the finite difference (FD) approximation of the Laplace operator followed by FFT [5, 12], which only gives solutions with accuracy of order $O\left(h^{2}\right)$ or $O\left(h^{4}\right)$ for the so-called 5-point or modified 9-point FD approximation, respectively ( $h$ is, of course, the size of the spacing distance of the sampling grid). Moreover, the 
computational cost of the ABIV algorithm is of order $O\left(N^{d} \log N\right)$, where $N$ is the number of grid points in each direction of a rectangular domain. This implies that the ABIV method is a fast algorithm. To describe the ABIV method, let us consider the domain $\Omega=(0,1)^{2}$ and Laplace's equation with its boundary conditions given by

$$
\begin{array}{rll}
\Delta u & =0 & (x, y) \in \Omega \\
u & =\phi_{1} & (x, y) \in[0,1] \times\{y=0\} \\
u & =\phi_{2} & (x, y) \in\{x=0\} \times[0,1] \\
u & =\phi_{3} & (x, y) \in[0,1] \times\{y=1\} \\
u & =\phi_{4} & (x, y) \in\{x=1\} \times[0,1] .
\end{array}
$$

One simplest analytic solution can be sought as

$$
\begin{aligned}
u(x, y)= & \sum_{k=1}^{\infty} b_{k}^{(1)} h_{k}(x, 1-y)+\sum_{k=1}^{\infty} b_{k}^{(2)} h_{k}(y, 1-x)+\sum_{k=1}^{\infty} b_{k}^{(3)} h_{k}(x, y)+ \\
& \sum_{k=1}^{\infty} b_{k}^{(4)} h_{k}(y, x)
\end{aligned}
$$

with

$$
h_{k}(x, y)=\sin k \pi x \frac{\sinh k \pi y}{\sinh k \pi} .
$$

The function $h_{k}(x, y)$ satisfies:

1. $\Delta h_{k}=0 \quad(x, y) \in \Omega$.

2. $h_{k}(x, y)$ vanishes on three sides of $\Omega$ i.e., on $x=0, x=1$ and $y=0$.

3. $\left\{h_{k}(x, 1)\right\}_{k=1}^{\infty}$ serves as a Fourier sine basis for $L^{2}([0,1])$.

4. $\left\{b_{k}^{(i)}\right\}$ are Fourier sine coefficients of $\phi_{i}$.

A mental picture of (17) can be depicted as four independent harmonic flows coming from each direction (see Figure 16).

However, in practice, $\phi_{i}$ 's are given as sampled data points on the boundary. The infinite sum in (17) is replaced by the sum of the first $N$ terms. Those coefficients are calculated via DST. Hence, how well the sum of the first $N$ terms approximate the true function, from which the boundary data are sampled, will directly affect the accuracy 


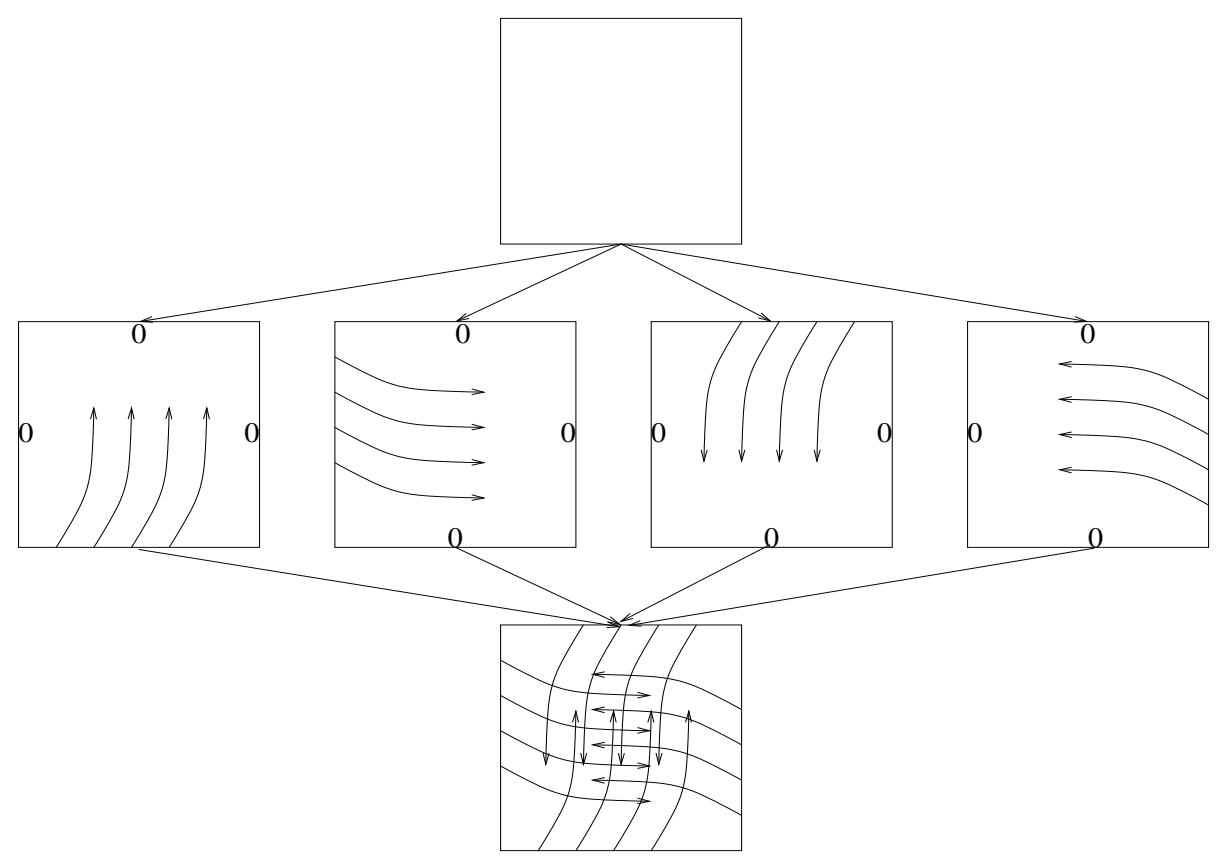

Figure 16: Each infinite sum in (17) is represented as a flow.

of the solution. One should notice that Fourier sine expansion is most suitable (efficient $N$ term approximation) for functions that vanish at two ends.

We next state the most practical version of the ABIV method for solving the system (16). Assume the continuity of the boundaries at four corners (i.e., $\phi_{1}(0)=\phi_{2}(0)=$ $\left.A, \phi_{2}(1)=\phi_{3}(0)=B, \phi_{3}(1)=\phi_{4}(1)=C, \phi_{4}(0)=\phi_{1}(1)=D\right)$. The ABIV algorithm proceeds as follows:

1. Seek a polynomial, $p(x, y)=a_{3} x y+a_{2} x+a_{1} y+a_{0}$, such that: $p(0,0)=A \quad p(0,1)=B \quad p(1,1)=C \quad p(1,0)=D$. 
2. Solve for $w$

$$
\begin{aligned}
& \Delta w=0 \quad(x, y) \in \Omega \\
& w=\phi_{1}-p \quad(x, y) \in[0,1] \times\{y=0\} \\
& w \quad=\phi_{2}-p \quad(x, y) \in\{x=0\} \times[0,1] \\
& w \quad=\phi_{3}-p \quad(x, y) \in[0,1] \times\{y=1\} \\
& w \quad=\phi_{4}-p \quad(x, y) \in\{x=1\} \times[0,1] .
\end{aligned}
$$

Note: $\phi_{i}-p$ vanishes at two ends. Hence the $N$ term approximation in (17) is suitable for $w$.

3. Set $u=p+w$.

For more complicated situations such as end value jumps at four corners or solutions with higher order accuracy, we refer the readers to their original papers $[1,4]$.

\section{References}

[1] A. Averbuch, M. Israeli, and L. Vozovoi. A fast Poisson solver of arbitrary order accuracy in rectangular regions. SIAM J. Sci. Comput., 19(3):933-952, 1998.

[2] B. Bialeck and A. Karageorghis. A Legendre spectral Galerkin method for the biharmonic Dirichlet problem. SIAM J. Sci. Comput., 22(5):1549-1569, 2000.

[3] P. Bjørstad. Fast numerical solution of the biharmonic Dirichlet problem on rectangles. SIAM J. Numer. Anal., 20(1):59-71, 1983.

[4] E. Braverman, M. Israeli, A. Averbuch, and L. Vozovoi. A fast 3D Poisson solver of arbitrary order accuracy. J. Comput. Phys., 144:109-136, 1998.

[5] W. L. Briggs and V. E. Henson. The DFT: An Owner's Manual for the Discrete Fourier Transform. SIAM, Philadelphia, PA, 1995.

[6] M. D. Buhmann. Radial basis functions. Acta Numerica, pages 1-38, 2000. 
[7] R. R. Coifman and Y. Meyer. Remarques sur l'analyse de Fourier à fenêtre. Comptes Rendus Acad. Sci. Paris, Série I, 312:259-261, 1991.

[8] N. A. Dodgson. Image resampling. Technical Report UCAM-CL-TR-261, University of Cambridge, 1992. ISSN: 1476-2986.

[9] R.L Hardy. Multiquadric equations of topographic and other irregular surfaces. Journal of Geophysical Research, 76:1905-1915, 1971.

[10] R.L Hardy and W.M Gopfert. Least squares prediction of gravity anomalies, geoidal undulations, and deflections of the vertical with multiquadric harmonic functions. Geophysical Research Letters, 2(10):423-427, 1975.

[11] R.L Hardy and S.A. Nelson. A multiquadric-biharmonic representation and approximation of disturbing potential. Geophysical Research Letters, 13(1):18-21, 1986.

[12] A. Iserles. A First Course in the Numerical Analysis of Differential Equations. Cambridge Texts in Applied Mathematics. Cambridge Univ. Press, New York, 1996.

[13] H. S. Malvar. Lapped transforms for efficient transform/subband coding. IEEE Trans. Acoust., Speech, Signal Process., 38:969-978, 1990.

[14] H. S. Malvar and D. H. Staelin. The LOT: transform coding without blocking effects. IEEE Trans. Acoust., Speech, Signal Process., 37:553-559, 1989.

[15] A. Mayo. The fast solution of Poisson's and the biharmonic equations on irregular regions. SIAM J. Numer. Anal., 21(2):285-299, 1984.

[16] C. A. Micchelli. Inerpolation of scattered data: distance matrices and conditionaly positive definite functions. Constr. Approx., 1:11-22, 1986.

[17] C. Rabut. Elementary $m$-harmonic cardinal B-splines. Numerical Algorithms, 2:39-62, 1992. 
[18] N. Saito and J.-F. Remy. A new local sine transform without overlaps: A combination of computational harmonic analysis and PDE. In M. A. Unser, A. Aldroubi, and A. F. Laine, editors, Wavelets: Applications in Signal and Image Processing X, volume Proc. SPIE 5207, pages 495-506, 2003.

[19] N. Saito and J.-F. Remy. The polyharmonic local sine transform: A new tool for local image analysis and synthesis without edge effect. Applied and Computational Harmonic Analysis, 20(1):41-73, 2006.

[20] M. Unser, D. Van De Ville, and T. Blu. Isotropic polyharmonic B-splines: Scaling functions and wavelets. IEEE Trans. Image Process., 14(11):1798-1813, November 2005 .

[21] G. Wahba. Spline Models for Observational Data, volume 59 of CBMS-NSF Regional Conference Series in Applied Mathematics. SIAM, Philadelphia, 1990.

[22] Z. Wang, A. C. Bovik, H. R. Sheikh, and E. P. Simoncelli. Image quality assessment: From error measurement to structural similarity. IEEE Trans. Image Process., 13(4):600-613, 2004.

[23] K. Yamatani and N. Saito. Improvement of DCT-based compression algorithms using Poisson's equation. IEEE Trans. Image Process., 15(12):3672-3689, 2006. 
Affiliation of authors:

Jucheng Zhao

Formerly Department of Mathematics, University of California, Davis, CA 95616

USA. Currently Prescio Consulting, LLC., Casa Grande, AZ 85230 USA.

Further author information: Email: jim@math.ucdavis.edu.

Naoki Saito

Department of Mathematics, University of California, Davis, CA 95616 USA.

Further author information: Email: saito@math.ucdavis.edu; WWW:

http://www.math.ucdavis.edu/ saito

Yi Wang

Department of Mathematics, Auburn University Montgomery, P.O. Box 244023, Montgomery, AL 36124 USA.

Further author information: Email: ywang2@ mail.aum.edu. 